\title{
Correction: Evidence-based scientific exercise guidelines for adults with spinal cord injury: an update and a new guideline
}

Kathleen A. Martin Ginis $\mathbb{C}^{1,2} \cdot$ Jan W. van der Scheer ${ }^{3} \cdot$ Amy E. Latimer-Cheung ${ }^{4} \cdot$ Andy Barrow $^{5} \cdot$ Chris Bourne $^{6}$. Peter Carruthers ${ }^{7} \cdot$ Marco Bernardi $^{8} \cdot$ David S. Ditor $^{9} \cdot$ Sonja Gaudet $^{10} \cdot$ Sonja de Groot $^{11} \cdot$ Keith C. Hayes $^{12}$. Audrey L. Hicks ${ }^{13}$. Christof A. Leicht ${ }^{3}$. Jan Lexell ${ }^{14}$ - Steven Macaluso ${ }^{15}$ - Patricia J. Manns ${ }^{16}$. Christopher B. McBride ${ }^{10} \cdot$ Vanessa K. Noonan $^{17} \cdot$ Pierre Pomerleau $^{18} \cdot$ James H. Rimmer $^{19} \cdot$ Robert B. Shaw $^{1}$. Brett Smith $^{20} \cdot$ Karen M. Smith ${ }^{4} \cdot$ John D. Steeves ${ }^{2} \cdot$ Dot Tussler $^{21} \cdot$ Christopher R. West $^{1} \cdot$ Dalton L. Wolfe ${ }^{22}$. Victoria L. Goosey-Tolfrey ${ }^{3}$

Published online: 4 October 2018

(c) International Spinal Cord Society 2018

Correction to: Spinal Cord (2018) 56:308-321 https://doi. org/10.1038/s41393-017-0017-3

Authors Victoria L. Goosey-Tolfrey and Karen M. Smith were listed under the incorrect affiliations at the time of publication.
Victoria L. Goosey-Tolfrey should have been affiliated with:

${ }^{3}$ Loughborough University, Loughborough, UK

Karen M. Smith should have been affiliated with:

${ }^{4}$ Queen's University, Kingston, Canada
Kathleen A. Martin Ginis

kathleen_martin.ginis@ubc.ca

University of British Columbia, Kelowna, Canada

ICORD, Vancouver, Canada

Loughborough University, Loughborough, UK

Queen's University, Kingston, Canada

5 Paralympian and Inspirational Speaker, London, UK

Spinal Cord Injury Ontario, Ottawa, Canada

Bromakin Wheelchairs, Loughborough, UK

8 Sapienza University of Rome, Rome, Italy

9 Brock University, St. Catharines, Canada

10 Spinal Cord Injury British Columbia, Vancouver, Canada

11 University of Groningen, Groningen, The Netherlands
12 Ontario Neurotrauma Foundation, Toronto, Canada

13 McMaster University, Hamilton, Canada

14 Lund University, Lund, Sweden

15 Western University, London, Canada

16 University of Alberta, Edmonton, Canada

17 Rick Hansen Institute, Vancouver, Canada

18 Institut de Réadaptation en Déficience Physique de Québec, Ville de Québec, Canada

19 University of Alabama at Birmingham, Alabama, USA

20 University of Birmingham, Birmingham, UK

21 National Spinal Injuries Centre, Stoke Mandeville Hospital, Aylesbury, UK

22 Parkwood Institute, Lawson Health Research Institute, London, Canada 